\title{
An Evolutionary Function Approximation Approach to Compute Prediction in XCSF
}

\author{
Ali Hamzeh and Adel Rahmani \\ Department of Computer Engineering, \\ Iran University of Science and Technology, Narmak, Tehran, Iran \\ \{hamzeh, rahmani\} @iust.ac.ir
}

\begin{abstract}
XCSF is a new extension to XCS that is developed to extend XCS's reward calculation capability via computing. This new feature is called computable prediction. The first version of XCSF tries to find the most appropriate equation to compute each classifier's reward using a weight update mechanism. In this paper, we try to propose a new evolutionary mechanism to compute these equations using genetic algorithms.
\end{abstract}

\section{Introduction}

$\mathrm{XCSF}$ [1] is a new extension to XCS [2] which extends ability of learning classifier systems to enable them to compute environmental rewards instead of memorizing them in all situations. In [1], author proposed XCSF and designed it to compute a linear piece-wise approximation for payoff function. This function is approximated using linear equations, which consist of two real coefficients that are called weights. These weights are updated using Widrow-Hoff update rule [3]. With respect to described issues in [4] to update these weights, we try to employ Genetic Algorithms [5] to approximate desired payoff functions. The rest of this paper is organized as follows: in the next section, we describe XCSF in brief, and then some relevant works on XCSF are presented. Then we describe our proposed method and our benchmark problems. At last, new method's results are presented and discussed.

\section{$2 \mathrm{XCSF}$ in Brief}

XCSF [1] is a model of learning classifier system that extends the typical concept of classifiers through the introduction of a computed classifier prediction. To develop XCSF, XCS has to be modified in three respects: (i) classifier conditions are extended for numerical inputs, as done in XCSI [6]; (ii) classifiers are extended with a vector of weights $\vec{w}$, that are used to compute the classifier prediction; (iii) The original update of the classifier prediction must be modified so that the weights are updated instead of the classifier prediction. These three modifications result in a version of XCS, XCSF [1] that maps numerical inputs into actions [7] with an associated calculated prediction.

Classifiers: In XCSF, classifiers consist of a condition, an action, and four main parameters. The condition specifies which input states the classifier matches; as in 
$\mathrm{XCSI}[6]$, it is represented by a concatenation of interval predicates, int $t_{i}=\left(l_{i} ; u_{i}\right)$, where $l_{i}$ ("lower") and $u_{i}$ ("upper") are integers, though they might be also real. The action specifies the action for which the payoff is predicted. The four parameters are:

(i) The weight vector $w$, used to compute the classifier prediction as a function of the current input; (ii) The prediction error $\mathcal{E}$, that estimates the error affecting the classifier prediction; (iii) The fitness $F$ that estimates the accuracy of the classifier prediction; (iv) The numerosity num, a counter used to represent different copies of the same classifier.

Performance Component: XCSF works as XCS. At each time step $t$, XCSF builds a match set $[\mathrm{M}]$ containing the classifiers in the population $[\mathrm{P}]$ whose condition matches the current sensory input $s_{t}$; if [M] contains less than $\theta_{\text {mи }}$ actions, covering takes place and creates a new classifier that matches the current inputs and has a random action. Each interval predicate $i n t_{i}=\left(l_{i} ; u_{i}\right)$ in the condition of a covering classifier is generated as $l_{i}=s_{t}(i)-\operatorname{rand} d_{l}\left(r_{0}\right)$, and $u_{i}=s_{t}(i)+\operatorname{rand}_{l}\left(r_{0}\right)$, where $s_{t}(i)$ is the input value of state $s_{t}$ matched by the interval predicated $i n_{i}$, and the function rand $_{l}\left(r_{0}\right)$ generates a random integer in the interval $\left[0 ; r_{0}\right]$ with $r_{0}$ fixed integer. The weight

vector $\vec{w}$ of covering classifiers is initialized with zero values; all the other parameters are initialized as in XCS [8]. For each action $a_{i}$ in [M], XCSF computes the system prediction which estimates the payoff that XCSF expects when action $a_{i}$ is performed. As in XCS, in XCSF the system prediction of action $a$ is computed by the fitness-weighted average of all matching classifiers that specify action $a$. However, in contrast with XCS, in XCSF the classifier prediction is computed as a function of the current state $s_{t}$ and the classifier vector weight $\vec{w}$. Following a notation similar to [8], the system prediction for action $a$ in state $s_{t}, P\left(s_{t} ; a\right)$, is defined as:

$$
P\left(s_{t}, a\right)=\frac{\sum_{c l \in[M] a} c l . p\left(s_{t}\right) \times c l . F}{\sum_{c l \in[M] l a} c l . F}
$$

Where $c l$ is a classifier, $[\mathrm{M}]_{\mathrm{a}}$ represents the subset of classifiers in [M] with action $a$, $c l . F$ is the fitness of $c l ; c l . p\left(s_{t}\right)$ is the prediction of $c l$ computed in the state $s_{t}$. In particular, $c l . p\left(s_{t}\right)$ is computed as:

$$
c l . p\left(s_{t}\right)=c l . w_{0} \times x_{0}+\sum_{t>0} c l . w_{i} \times s_{t}(i)
$$

Where $c l . w_{i}$ is the weight $w_{i}$ of $c l$ and $x_{0}$ is a constant input. The values of $P\left(s_{t} ; a\right)$ form the prediction array. Next, XCSF selects an action to perform. The classifiers in $[\mathrm{M}]$ that advocate the selected action are put in the current action set [A]; the selected action is sent to the environment and a reward $r$ is returned to the system together with the next input state $s_{t+l}$. Note that when XCSF is applied to function approximation problems, as in this paper, there is only one dummy action that the system can perform which has not actual effect on the environment [6].

Reinforcement Component: XCSF uses the incoming reward $r$ to update the parameters of classifiers in action set [A]. First, the reward $r$ is used to update the 
weight vector $\vec{w}$ using a modified delta rule [3] as follows For each classifier $c l \in[\mathrm{A}]$, each weight $c l . w_{i}$ is adjusted by a quantity $\Delta w_{i}$ computed as:

$$
\Delta w_{i}=\frac{\eta}{\left|x_{t}-1\right|^{2}}\left(r-c l \cdot p\left(s_{t-1}\right)\right) x_{t-1}(i)
$$

Where $\eta$ is the correction rate and $\vec{x}_{t-1}$ is defined as the input state vector $\left|\vec{x}_{t-1}\right|^{2} S_{t-1}$ augmented by a constant $x_{0}$ (i.e. $\left.x_{t-1}=\left\langle x_{0}, s_{t-1}(1), \vec{s}_{t-1}(2), \ldots, s_{t-1}(n)\right\rangle\right)$ and is the norm of vector $\vec{x}_{t-1}$ for further details refer to [1] The values $\Delta w_{i}$ are used to update the weights of classifier $c l$ as:

$$
c l . w_{i} \leftarrow c l . w_{i}+\Delta w_{i}
$$

A classifier in XCSF basically represents a perceptron with a linear activation function [9] which is applied only on the inputs that match the classifier condition. Then the prediction error $\mathcal{E}$ is updated as:

$$
c l . \varepsilon \leftarrow c l . \varepsilon+\beta\left(\left|r-c l . p\left(s_{t-1}\right)\right|-c l . \varepsilon\right)
$$

Classifier fitness is updated as in XCS. First, the raw accuracy $\kappa$ of the classifiers in $[\mathrm{A}]$ is computed as follows.

$$
\kappa= \begin{cases}1 & \text { if } \varepsilon \leq \varepsilon_{0} \\ \alpha\left(\varepsilon / \varepsilon_{0}\right)^{-v} & \text { otherwise. }\end{cases}
$$

The raw accuracy is used to calculate the relative accuracy $\varepsilon_{0}$ as

$$
\kappa^{\prime}=\frac{\kappa \times \text { num }}{\sum_{c l[[A]}(\text { cl. } . \kappa \times \text { cl.num })}
$$

Where $c l . \kappa$ is the raw accuracy of classifier $c l$; cl.num is the numerosity of classifier $c l$. Finally, the relative accuracy $\boldsymbol{K}^{\prime}$ is used to update the classifier fitness as: $F \leftarrow F+\beta\left(\kappa^{\prime}-F\right)$.

Discovery Component: The genetic algorithm in XCSF works as in XCSI [6]. The genetic algorithm is applied to classifiers in [A]. It selects two classifiers with probability proportional to their fitness, copies them, and with probability $\chi$ performs crossover on the copies; then, with probability $\mu$ it mutates each allele. Crossover and mutation work as in XCSI.

\section{Relevant Works on XCSF}

In [4], the authors study generalization in XCSF and introduce several different approaches to improve the generalization capabilities of XCSF. At first, they present experimental evidence showing that while XCSF always evolves accurate approximations, the types of generalizations evolved can be influenced by the input range. The presented results show that when the inputs are limited to small values, 
XCSF evolves (accurate) piecewise linear approximations, as expected. But when the input range includes large values, XCSF does not fully exploit its learning capabilities and tends to evolve (accurate) piecewise constant approximations.

In [11] authors take XCSF one step further and apply it to typical reinforcement learning problems involving delayed rewards. In essence, they use XCSF as a method of generalized (linear) reinforcement learning to evolve piecewise linear approximations of the payoff surfaces of typical multistep problems. Achieved results show that XCSF can easily evolve optimal and near optimal solutions for problems introduced in the literature to test linear reinforcement learning methods.

Authors show in [12] that how XCSF can be easily extended to allow polynomial approximations. They test the extended version of XCSF on various approximation problems and shows that quadratic/cubic approximations can be used to significantly improve XCSF's generalization capabilities.

Moreover, in [13] authors apply XCSF to the learning of Boolean functions. The presented results show that XCS with computable linear prediction performs optimally in typical Boolean domains and it allows more compact solutions evolving classifiers that are more general compared with XCS.

\section{XCSF with Evolutionary Function Approximation Approach}

In this section, we describe overall architecture and implementation details of the proposed extension to XCSF. As we described in previous section, Widrow-Hoff update rule encounter some difficulties in certain range of input variables. In [4], some solutions to solve these problems are proposed. We are trying to propose a new solution which has no need to any weight update process to approximate desired payoff functions. This new system is called XCSF-G. XCSF-G's overall architecture is the same as XCSF with some minor modifications. However, the major difference between XCSF-G and traditional XCSF is classifier's structure. Classifier's structure is XCSF-G is modified to support GA function approximation capability.

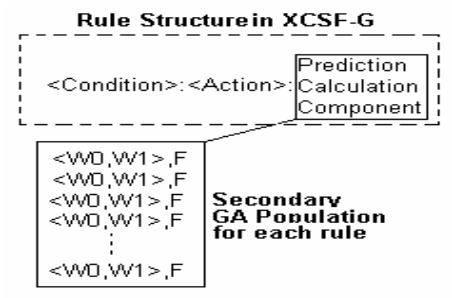

Fig. 1. Structure of XCSF-G's rule

In this type of rule, prediction is calculated by a secondary population of chromosomes which is allocated for each rule separately.

\subsection{Evolutionary Prediction Calculation Procedure}

In this section we describe important points to understand genetic algorithm-based prediction calculation method: 


\subsubsection{Calculate Prediction}

To illustrate this, we describe structure of secondary population's chromosomes.

These chromosomes contain two real numbers. These numbers are imagined as $w_{0}$ and $w_{1}$ in XCSF for each rule. To calculate prediction for each rule, we follow this procedure: At first, we choose best chromosome in the secondary population with respect to fitness and calculate the prediction using equation 7 :

$$
w_{0}+w_{1} x_{1}
$$

where $w_{0}$ and $w_{1}$ are evolved genes in the best chromosome of the population and $x_{1}$ is environmental input for the current state.

\subsubsection{Secondary Population's Architecture}

As we mentioned in previous section, to calculate prediction for each rule, we must choose best chromosome of its secondary population with respect to its fitness. So fitness calculation of the secondary population is one of the most important parts of XCSF-G. In fitness calculation routine, the most important goal is to select the individual with lowest error rate in previous prediction estimation epochs. So, in $\mathrm{XCSF}-\mathrm{G}$ fitness calculation is done using rule's prediction error. In every trial of XCSF-G's life cycle, a tuple is made of environmental input and reward; we call this tuple an Estimation Twin (ET). ETs are listed and memorized for each fired rule which was involved in reward gathering procedure. When number of stored ET's of a specified rule reaches a predefined threshold $n_{E T}$, then fitness calculation of secondary populations of this rule begins as follows:

- For each chromosome, estimated output for given input is calculated using equation 7 separately and overall error is calculated using equation:

$$
E_{j}=\frac{\sum_{i=1}^{n}\left[G_{j}\left(x_{i}\right)-P\left(x_{i}\right)\right]^{2}}{n_{E T}}
$$

Where $G_{j}\left(x_{i}\right)$ is estimated value of $j$ 'Th chromosome for $x_{i}$ and $\mathrm{P}\left(\mathrm{x}_{\mathrm{i}}\right)$ is environmental reward for the same input. Then fitness for $j$ 'Th chromosome is calculated using:

$$
F_{j}=e^{-E_{j}}
$$

- After calculating fitness for all chromosomes, mating pool for secondary population is constructed using tournament selection [10] with tour size of two.

- After selecting parents from mating pool randomly, one offspring is generated using parent's genes with respect to their calculated fitness.

$$
w_{0}^{c}=\frac{f^{p 1} w_{0}^{p 1}+f^{p 2} w_{0}^{p 2}}{f^{p 1}+f^{p 2}}, w_{1}^{c}=\frac{f^{p 1} w_{1}^{p 1}+f^{p 2} w_{1}^{p 2}}{f^{p 1}+f^{p 2}}
$$

- Above procedure is repeated until next generation of secondary population is constructed completely.

\subsubsection{Initial Population Initialization}

After producing new rule in XCSF-G, secondary population for this rule must be initialized. This population is initialized randomly and their fitness's are set to a random number in $[0 ; 0.1)$ interval. 


\section{Design of Experiments}

All the experiments discussed in this paper involve single step problems and are performed following the standard design used in the literature $[4,5]$. In each experiment XCSF has to learn to approximate a target function $f(x)$; each experiment consists of a number of problems that $\mathrm{XCSF}(-\mathrm{G})$ must solve. For each problem, an example $\langle x, f(x)\rangle$ of the target function $f(x)$ is randomly selected; $x$ is input to XCSF(-

G) whom computes the approximated value $\hat{f(x)}$ has the expected payoff of the only available dummy action; the action is virtually performed (the action has no actual effect), and $\mathrm{XCSF}(-\mathrm{G})$ receives a reward equal to $f(x)$. Each problem is either a learning problem or a test problem. In learning problems, the genetic algorithm is enabled while it is turned off during test problems. The covering operator is always enabled, but operates only if needed. XCSF performance is measured as the accuracy of the evolved approximation $\hat{f(x)}$ with respect to the target function $f(x)$. To evaluate the evolved approximation $f(x)$ we measure the mean absolute error, MAE:

$$
M A E=\frac{1}{n} \sum_{x}|f(x)-\hat{f}(x)|
$$

Where $\mathrm{n}$ is the number of points for which $f(x)$ is defined. In particular we use the average MAE over the performed experiments, dubbed MAE.

\section{Experimental Results}

We now compare XCSF with XCSF-G. For this purpose, we have considered problems from $\sin$ family. This family is very flexible. It means that we can change range of input variable and function slope with very simple parameter setting. This feature helps us to analyze our solution's weaknesses and advantages with respect to discussed issues in [4]. This family of problems is represented using equation 13:

$$
f(x)=\sin (\operatorname{Slp} * \pi(U p-x) / 50)
$$

where $S l p$ determines function's slope and $U p$ determines range of input variable. In this paper, $S l p$ and $U p$ are chosen from table 1:

Table 1. Experienced values for $U p$ and $S l p$

\begin{tabular}{|c|c|c|c|}
\hline \multirow{2}{*}{$\boldsymbol{U} \boldsymbol{p}$} & \multicolumn{3}{|c|}{$\boldsymbol{S l p}$} \\
\cline { 2 - 4 } & 1 & 2 & 5 \\
\hline 50 & PR1 & PR2 & PR3 \\
\hline 1050 & PR4 & PR5 & PR6 \\
\hline
\end{tabular}

This table is interpreted as follows: the problem number determines $S l p$ and $U p$, for example $P R I$ is presented using equation 13 and $P R 5$ is determined with equation 14.

$$
\begin{gathered}
P R 1(x)=\sin (\pi(50-x) / 50) \\
P R 5(x)=\sin (2 \pi(1050-x) / 50)
\end{gathered}
$$




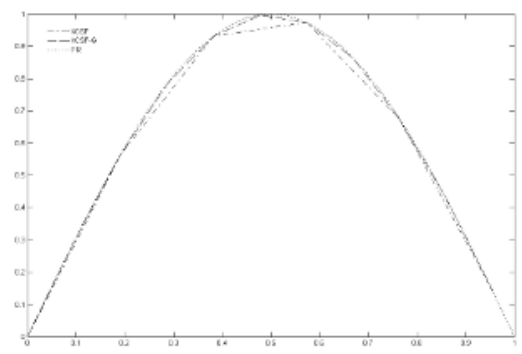

(a)

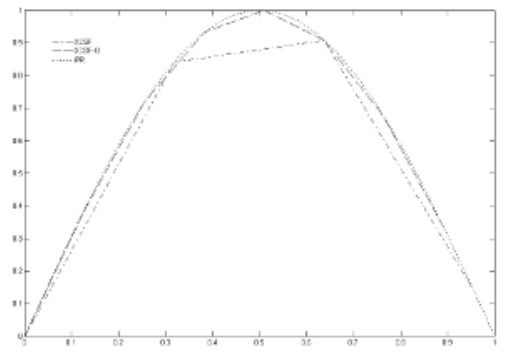

(b)

Fig. 2. XCSF (dashed-dot), XCSFG (dashed) in PR1 (a) and PR4 (b), PRs are dotted lines.

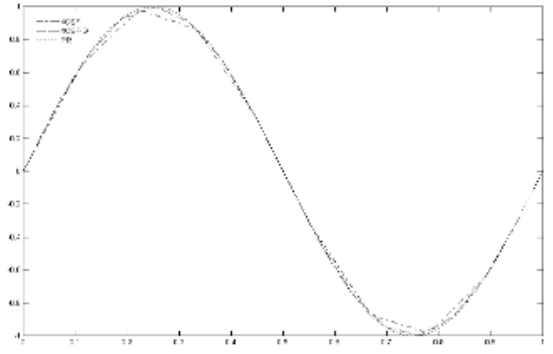

(a)

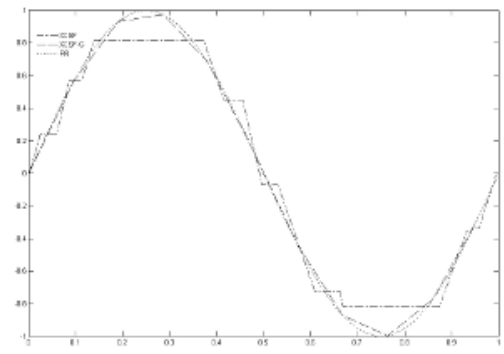

(b)

Fig. 3. XCSF (dashed- dot), XCSFG (dashed) in PR2 (a) and PR5 (b), PRs are dotted lines

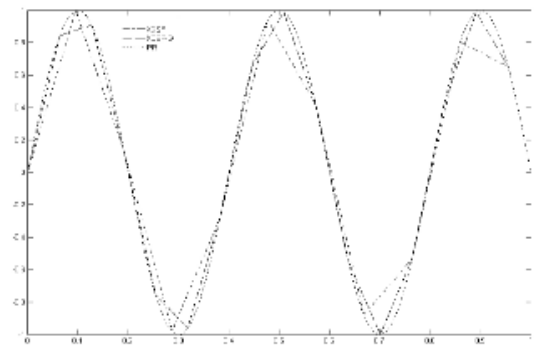

(a)

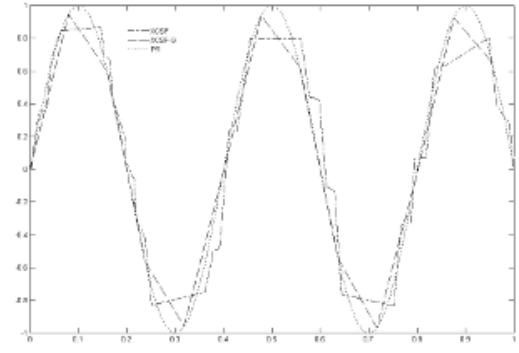

(b)

Fig. 4. XCSF (dashed line), XCSFG (dashed) in PR3 (a) and PR6 (b), PRs are dotted lines

It is notable that input variable $x$ is chosen from the interval $[U p-50, U p]$ for all problems. For the experiments discussed here we always use the same parameter settings: $N=1000 ; \beta=0.2 ; \alpha=0.1 ; v=5 ; \chi=0.8, \mu=0.04, \theta_{n m a}=1, \theta_{d e l}=50 ; \theta_{G A}=$ $50 ; \delta=0: 1$; GA-subsumption is on with $\theta_{\text {sub }}=50$; while action-set subsumption is off. Secondary genetic population in XCSF-G's size is equal to 50 and $n_{E T}=10$. Below 
XCSF and XCSF-G are compared in described problems and their approximated functions are shown in figure 2 to 4 . These figures are drawn using the following procedure. Both XCSF and XCSF-G are allowed to run for 1500 learning trials then all of the 300 selected values which are uniformly distributed in the learning interval (e.g. $[0,50])$ are fed to both of them and this procedure is repeated 50 times and average values for this 50 runs are plotted in figures 2 to 4 .

\section{Discussion}

As it was mentioned in previous sections, the numerical parameter to evaluate XCSF and XCSF-G is MAE that is calculated using equation 12. This value is calculated using 300 uniformly distributed points between $[U p-50, U p]$. These values are shown in table 2 .

Table 2. MAE values for XCSF and XCSF-G

\begin{tabular}{|c|c|c|}
\hline Problem Number & XCSF's MAE & XCSF-G's MAE \\
\hline $\boldsymbol{P R I}$ & 0.015 & 0.009 \\
\hline $\boldsymbol{P R 2}$ & 0.019 & 0.014 \\
\hline $\boldsymbol{P R 3}$ & 0.02 & 0.04 \\
\hline $\boldsymbol{P R 4}$ & 0.05 & 0.01 \\
\hline $\boldsymbol{P R 5}$ & 0.15 & 0.025 \\
\hline $\boldsymbol{P R 6}$ & 0.4 & 0.06 \\
\hline
\end{tabular}

It is clear that XCSF and XCSF-G both can solve the set of problems with small input values (such as $P R 1, P R 2$ and $P R 3$ ). But an interesting issue is that when the desired function to approximate becomes more complex, XCSF-G seems to be less accurate than XCSF and becomes slightly weaker to approximate complex functions such as $P R 3$. However, the most interesting issue arises when input range changes from small real numbers to big ones (e.g $U p$ is set to 1050 instead of 50). In this set of problems XCSF's performance decreases dramatically as described in [4]. This issue is obvious by only looking to proposed approximation of XCSF for PR4 to PR6. it is clear that XCSF proposed a piece-wise constant approximation for these problems as discussed in [4] and table 2 confirms this idea. XCSF's $M A E$ becomes larger in PR4-6 than their similar problems in PR1-3, but XCSF-G's performance just slightly changes and is significantly better than XCSF's performance.

\section{References}

1. Wilson, S. W. (2002). Classifiers that approximate functions. Journal of Natural Computating 1 (2-3), 211-234.

2. Wilson, S. W. (1995). Classifier Fitness Based on Accuracy. Evolutionary Computation 3.

3. Widrow, B. and M. E. Hoff (1988). Adaptive Switching Circuits, Chapter Neurocomputing: Foundation of Research, pp. 126-134. Cambridge: The MIT Press.

4. Lanzi, P. L., D. Loiacono, S. W. Wilson, and D. E. Goldberg (2005). Generalization in the XCSF Classifier System: Analysis, Improvement, and Extension. Technical Report 2005012, Illinois Genetic Algorithms Laboratory. 
5. Holland, J. H. (1975). Adaptation in Natural and Artificial Systems. Ann Arbor: University of Michigan Press. Republished by the MIT press, 1992.

6. Wilson, S. W. (2001). Function approximation with a classifier system. In proceedings of the Genetic and Evolutionary Computation Conference (GECCO-2001), San Francisco, California, USA, pp. 974-981. Morgan Kaufmann.

7. Wilson, S. W. (2004). Classifier systems for continuous payoff environments. In Genetic and Evolutionary Computation -GECCO-2004, Part II, Volume 3103 of Lecture Notes in Computer Science, Seattle, WA, USA, pp. 824-835. Springer-Verlag.

8. Butz, M. V. and S. W. Wilson (2002). An algorithmic description of XCS. Journal of Soft Computing 6 (3-4), 144-153.

9. Haykin, S. (1998). Neural Networks: A Comprehensive Foundation. Prentice Hall PTR.

10. Butz, M. V., K. Sastry, and D. E. Goldberg (2003). Tournament selection: Stable fitness pressure in XCS. In Genetic and Evolutionary Computation - GECCO-2003, Volume 2724 of LNCS, Chicago, pp. 1857-1869. Springer-Verlag.

11. Lanzi, P. L., D. Loiacono, S. W. Wilson, and D. E. Goldberg (2005). XCS with Computable Prediction in Multistep Environments. Technical Report 2005008, Illinois Genetic Algorithms Laboratory.

12. Lanzi, P. L., D. Loiacono, S. W. Wilson, and D. E. Goldberg (2005). Extending XCSF Beyond Linear Approximation. Illinois Genetic Algorithms Laboratory University of Illinois at Urbana-Champaign.

13. Lanzi, P. L., D. Loiacono, S. W. Wilson, and D. E. Goldberg (2005). XCS with Computable Prediction for the Learning of Boolean Functions. Technical Report 2005007, Illinois Genetic Algorithms Laboratory. 